\title{
COMPARATIVE CFD ANALYSIS OF AIRFOILS FOR UNMANNED AERIAL VEHICLES
}

\author{
Anjali Balani ${ }^{1}$, Tarun Yadav ${ }^{2}$, Appasaheb Malagaudanavar ${ }^{3}$ \\ ${ }^{I}$ Student, University of Michigan, Ann Arbor \\ ${ }^{2}$ Manager, AURDC, Hindustan Aeronautics Limited, Nasik Division, India \\ ${ }^{3}$ Addl General Manager, AURDC, Hindustan Aeronautics Limited, Nasik Division, India
}

\begin{abstract}
In recent years, Unmanned Aerial Vehicles (UAVs) have become an important constituent of the defence aircraft industry internationally. They are even preferred over conventional fighter aircraft in certain circumstances as they can be controlled remotely and have an endurance of approximately 24 to 48 hours. As in the case for most aircraft, wing profiles or airfoils have a large influence on the lift and efficiency of UAV wing structures too. Most current research focuses on comparison and analysis of various airfoils. Our study deals with the selection of a suitable airfoil for use in a UAV wing structure. We will accomplish this by selecting an available airfoil and then comparing it to an airfoil currently employed in a successful UAV.

Cambered airfoils which provide lift even at low or zero angles of attack are usually used in UAV wing structures. Based on this and other basic requirements for UAV wing structures, weused NACA 4415 airfoil for comparison with NASA LRN 1015 airfoil currently employed in the Northrop Grumman RQ-4 Global Hawk. Using Computational Fluid Analysis (CFD) we simulated the airflow about the aforementioned airfoils at Mach numbers and altitudes typical to UAVs. We thenobtained the values and trends of values for the coefficients of lift, drag and pitching moment for both airfoils. Key similarities were found between the lift drag and pitching coefficient characteristics and trends of these characteristics leading us to preliminarily conclude that NACA 4415 may be used in a UAV. Hereupon, further $3 D$ CFD analysis or wind tunnel experimentation with a 3D wing model may be undertaken to confirm this choice.
\end{abstract}

Keywords: Unmanned Aerial Vehicle, Airfoil, Lift theory, Lift, Drag, Pitching Moment,

\section{INTRODUCTION}

\subsection{Unmanned Aerial Vehicles}

An important class of airplanes that uses airfoils is Unmanned Aerial Vehicles (UAVs). These are aircraft that are often employed for surveillance and to conduct airstrikes. The key difference between UAVs and fighter aircraft is that UAVs are operated remotely and do not require a pilot. They are designed merely to spy on targets and to conduct targeted remote airstrikes on specific targets usually in war zones. These aircraft are also employed in reconnaissance missions where they have to stay afloat for a long time. As they are operated remotely, they are never used in active combat. Most UAVs fly at altitudes of up to about 19kilometers and at Mach numbers of 0.6.

\subsection{Airfoils and Airfoil Geometry}

The wing structures of UAVs are especially important components of the design as they provide most of the lift to the aircraft and hold most control surfaces. The cross sections of these wing structures which are responsible for lift and drag generation are known as airfoils.

By definition, an airfoil is the cross section of a body which when placed in an airstream produces a useful aerodynamic force.The most common use of airfoils is in the design of cross sections of airplane wings, propeller blades, turbine blades and hydrofoils.

The geometry of every airfoil can be described by certain terms such as Leading edge, Trailing edge, Chord Length, Camber, Mean Camber line, Thickness and Angle of Attack.

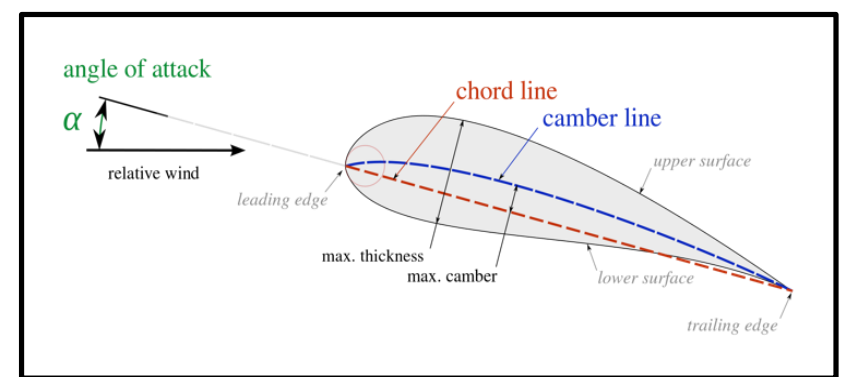

Fig -1: Airfoil Terminology

\subsection{Airfoil Lift Theories}

There are multiple theories that explain lift. The most common theory is the "Equal Transit" theory which explains that since the upper surface of an airfoil is longer than the lower surface, the air molecules at the top move with higher velocity to meet the particles travelling at the lower surface at the trailing edge. The difference in velocities produces difference in pressures which in turn produces lift. This was, however, proven to be incorrect.[1] 
Today, the two most accepted explanations are those that employ either Bernoulli's theorem or Newton's laws to explain lift. Although proponents of both explanations are in constant debate over the veracity of the others claims, both may be considered reasonable explanations for lift.

Bernoulli's theorem may be employed to explain lift as a result of pressure differences along the wing. On the other hand, Newton's laws explain lift as an action reaction pair of forces (the lift is produced due to the force exerted by the deflected gas). [2]

\subsection{NACA Airfoils}

Some of the most commonly used airfoils were designed by the National Advisory Committee for Aeronautics (NACA now NASA). These airfoils have been split into multiple series. One of these series is the 4-series, for example NACA 2214. The numbers in the name of NACA 4 series airfoil describe its geometry.

- The first number signifies the maximum camber as a percent of chord.

- The second number multiplied by 10 signifies the position of maximum camber as a percent of chord.

- The last two numbers signify the thickness as a percent of chord.

So using our example, if a NACA 2214 airfoil has chord of $1 \mathrm{~m}$, the maximum camber is $0.02 \mathrm{~m}$ located at $0.4 \mathrm{~m}$ with an airfoil thickness of $0.12 \mathrm{~m}$.

Airfoil geometry is extremely important as certain variables such as lift, drag polar and stability at the required Mach and Reynolds numbers depend on this geometry. When designing aircraft such as UAVs it is important to optimize the values for variables such as lift and drag by manipulating independent variables such as angle of attack in the conditions the aircraft is meant to operate in. Sometimes wind tunnel tests are conducted to obtain and compare the values for these variables. However, usually constructing models and doing physical tests is expensive in terms of time and resources employed. So often in the preliminary stages of design, airfoils are selected on the basis of mathematical calculations and simulations as a part of computational fluid dynamics (CFD).

\subsection{Computational Fluid Dynamics}

Computational fluid dynamics is a computerized method of study of airflows around bodies. As a part of this method a $2 \mathrm{D}$ or $3 \mathrm{D}$ structure is created and divided into numerous small parts known as cells. All the cells in the structure make up a mesh. Using constants such as pressure or Reynolds number of airflow that are inputs to the system, the effect of the airflow is calculated on each individual cell at a time and then the overall effect is summed up to give us the estimated values for lift, drag and pressure coefficients. The simulations allow us to change parameters and test multiple cases of the same problem in a short period of time.

\section{LITERATURE SURVEY}

2D NACA airfoils were researched extensively in the literature survey. Şahinet al [3]conducted experiments and simulated air flow under identical conditions using a wind tunnel and CFD respectively and concluded that both results were in close accordance with each other. Eleni et al [4] simulated the flow over a NACA 0012 airfoil through CFD using multiple turbulence models and compared the results obtained with experimentally obtained data to ascertain the accuracy of each model. It was concluded that the k- $\omega 2$ equation turbulence model was most appropriate for these conditions. It was concluded that a lot of the studies done on NACA airfoils were related to optimizing certain conditions for their use. In each study a certain independent variable such as angle of attack or pressure coefficient would be altered keeping the rest constant. When the flow of fluid was simulated using CFD this yielded different values for lift, drag, coefficients of lift and drag etc. in each case and allowed the author to optimize that independent variable. Kevadiyaet al [5] compared and optimized angle of attack for a NACA 4412 airfoil under certain conditions. He kept all the other independent variables constant and simulated the airflow around the airfoil which yielded certain values of pressure coefficients. He concluded that for a NACA 4412 airfoil the optimum angle of attack was $12^{\circ}$. Gulzaret al [6] simulated air flow around a NACA 7420 airfoil using the Spalart- Allmaras model and concluded that an angle of attack of $5^{\circ}$ proved to be optimum for this airfoil. Maratheet al [7] worked on simulating airflow about a NACA 2204 airfoil through CFD at low Reynolds number. This airfoil was inspected for use in a Miniature Air Vehicle (MAV). Hossain et al [8] compared NACA 6409 and NACA 4412 by simulating the airflow around them using CFD. It was concluded on the basis of the individual lift to drag ratios that NACA 4412 was better than NACA 7420 under given conditions. Ahmed et al [9] simulated the flow around a NACA 0012 airfoil and further simulated flow around a modified NACA 0012 airfoil with flaps at different angles. It was concluded that higher flap angles generated higher lift along with higher drag and that the lift to drag ratio could be optimized using flaps.

Based on the research conducted as a part of the literature survey I decided to work on estimating the possibility of using an airfoil for a UAV. I accomplished this by simulating the airflow around NASA LRN 1015 the airfoil used in a Northrop Grumman Global hawk[10].

Following this, I similarly simulated the airfoil about a NACA 4 series airfoil chosen based on some rough requirements for the camber and thickness.

\section{GOVERNING EQUATIONS}

Computational fluid dynamics (CFD) relies on certain governing equations to simulate airflow. The Navier-Stokes equation is used to model the turbulence in a fluid as it flows around a body. The following equations together account for the Navier-Stokes equations in two dimensions: 
- Continuity

$$
\frac{\partial \rho}{\partial t}+\nabla(\rho V)=0
$$

- Momentum (x direction)

$$
\frac{\partial(\rho u)}{\partial t}+\nabla(\rho u V)=-\frac{\partial \rho}{\partial x}+\frac{\partial \tau_{x x}}{\partial x}+\frac{\partial \tau_{y x}}{\partial y}
$$

- $\quad$ Momentum (y direction)

$$
\frac{\partial(\rho v)}{\partial t}+\nabla(\rho v V)=-\frac{\partial \rho}{\partial y}+\frac{\partial \tau_{x y}}{\partial x}+\frac{\partial \tau_{y y}}{\partial y}
$$

The Navier-Stokes equations can be solved using multiple approaches. The approach I used to simulate airflow is the Reynolds Averaged Navier-Stokes (RANS) approach. In this method turbulent scales are modelled so that no eddies are resolved. It views the flow as a scalar quantity which can be represented as a sum of its mean and fluctuating components. The solving of this equation leads to the formation of a new term knows as Reynolds stress. It needs to be modelled to solve the RANS approach. This is usually done by using the Boussinesq assumption and the turbulence viscosity $\mu_{t}$.

$$
-\rho \overline{\mu_{i}{ }^{\prime} \mu_{j}{ }^{\prime}}=\mu_{t}\left(\frac{\partial \mu_{i}}{\partial x_{j}}+\frac{\partial \mu_{i}}{\partial x_{j}}\right)-\frac{2}{3}\left(\rho k+\mu_{t} \frac{\partial \mu_{k}}{\partial x_{k}}\right) \partial_{i j}
$$

The RANS approach was employed in the software ANSYS Fluent with a density based solver.

There are multiple turbulence models that are used to solve the RANS approach. The one I employed is the $k-\omega$ Turbulence Model. This is a two equation model which accounts.

\section{COMPUTATIONAL FLUID DYNAMICS SET}

\section{UP}

\subsection{Geometry}

Two airfoils were employed in this comparative study. NASA LRN 1015 was chosen as it is used in the Northrop Grumman RQ-4 Global Hawk, an Unmanned Aerial Vehicle successfully employed for surveillance and combat in war zones.

The NACA 4415 was chosen as a candidate for investigation into its possible use in a future Unmanned Aerial Vehicle due to its high camber and asymmetrical shape would generate high lift even at $0^{\circ}$ angle of attack. This is necessary in any aircraft that seeks to maximize lift. Additionally, its similarity in shape to NASA LRN 1015 which would possibly ensure similar fluid flow The geometries of airfoils NACA 4415 and NASA LRN 1015 were generated in ICEM software after getting coordinates from internet source (reference). Figure 2(a) and 2(b) shows the geometry of NASA LRN 1015 andNACA 4415 airfoils respectively.

\subsection{Mesh Generation}

A fine mesh of both airfoils NACA 4415 and NASA LRN 1015 were generated in ICEM CFD 15.0 software. Both meshes are structured mesh and consist of 17000 mesh elements. The y+ was considered 5 during mesh generation. This was considered in order to capture the exact viscous flow behavior and boundary layer across airfoil. Figure 2(c) and 2(d) shows the structured mesh of NASA LRN 1015 and NACA 4415 airfoils respectively.After meshing, CFD simulations in ANSYS FLUENT 15.0 were carried out on all grids to analyse the flow behaviour of various nozzles.

\subsection{Boundary Conditions}

The external flow analysis was carried out by running simulations in commercial software ANSYS Fluent 15.0 version. The dimensional units were set to SI in Fluent.

\subsection{Material Properties}

The fluid considered during the case was ideal gas. The flow of fluid was taken ascompressible flow, where the value of mach number varies from inlet/pressure farfield to outlet. The properties of ideal gas taken are given below:

- $\quad$ Density = Ideal gas density

- $\quad$ Viscosity = 1.7894 e-5 Kg/m-s

- $\quad$ Thermal conductivity $=0.0242 \mathrm{~W} / \mathrm{m} . \mathrm{K}$

- $\quad$ Specific Heat capacity $(\mathrm{Cp})=1006.43 \mathrm{~J} / \mathrm{Kg} . \mathrm{K}$

- $\quad$ Molecular weight $=28.966 \mathrm{~kg} / \mathrm{kgmol}$.

The operating pressure was set to 0 Pascal during flow analysis. The other parameters like external air flow and gravitywere not taken into account. Following boundary conditions were set before running the simulation:

\subsection{Pressure Far Field}

The gauge pressure was set to 5529 Pascal. The Mach number was set to $0.6 \mathrm{M}$. Temperature was set to $216.5 \mathrm{~K}$.

Outlet: The outlet was taken as pressure outlet, where gauge pressure was set to 0 Pascal and backflow temperature was set to $0 \mathrm{~K}$.

Wall: The airfoil was considered as a stationary wall with no slip condition

Control Setup and Discretization: The Density based solver with Spalart Allmarasturbulence model and energy equation on was considered during simulations. The under relaxation factors were considered as default value. In solution methods, the implicit formulation with Roe-FDS flux type was set. The Green Gauss node based with second order upwind discretization and modified turbulent viscosity was considered. 


\subsection{Initialization}

The solution was initialized with standard initialization. The convergence criteria was set to 10-6.

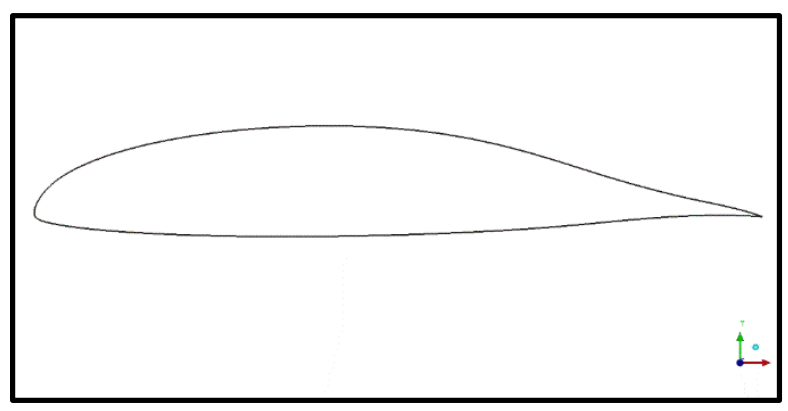

Fig -2(a):NASA LRN 1015

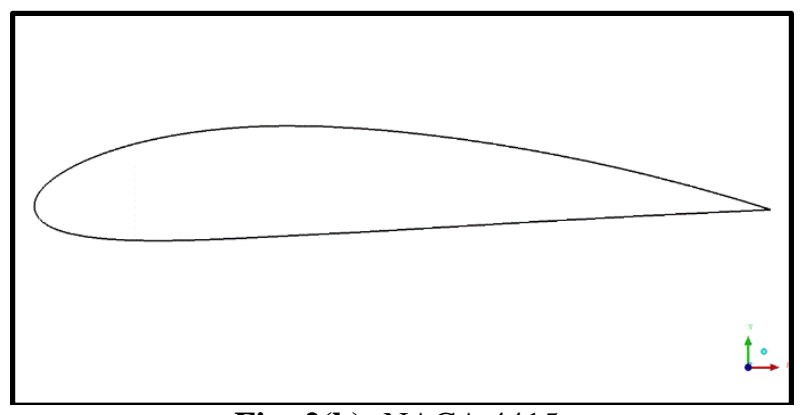

Fig -2(b): NACA 4415

Computational Fluid dynamics was used in my research to study the fluid flow around the two airfoils chosen. This method involved multiple steps. After the initial plotting and drawing of the airfoils was completed, the object to be analyzed was discretized via a mesh. Mesh formation by discretization of the domain into a large number of subdomains was accomplished.

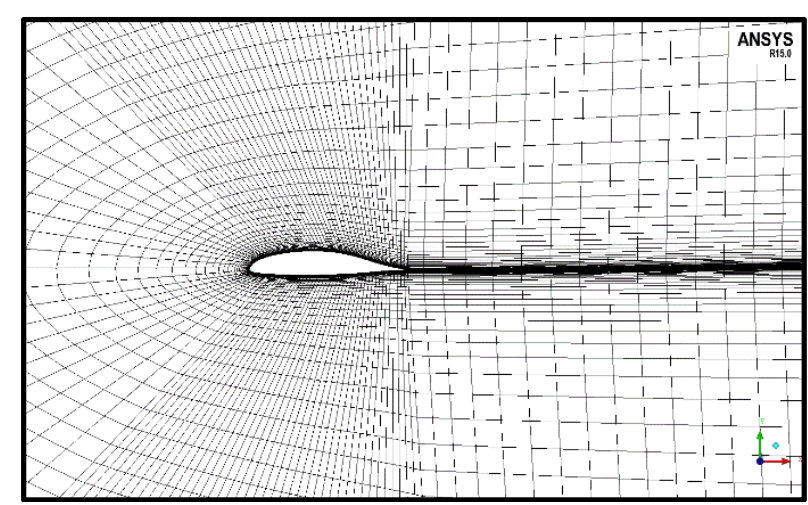

Fig -2(c): NACA 1015

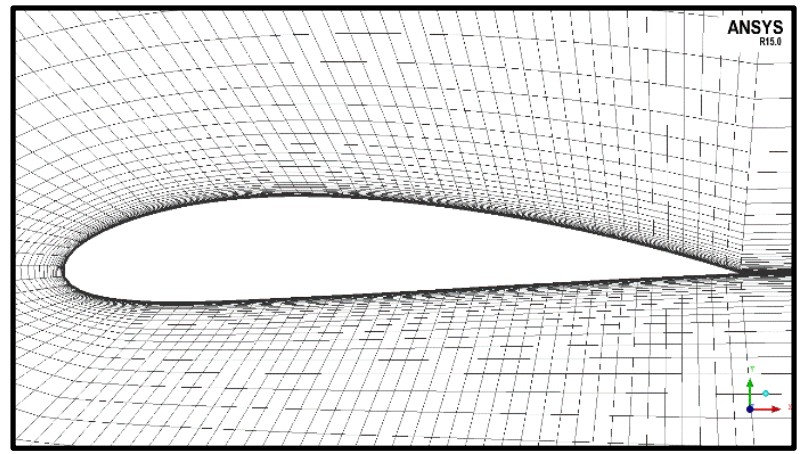

Fig -2(d): NACA 4415

A number of iterations were run on this setup to get the convergence and the values for coefficients of moment, lift, drag and were found to converge as shown below in charts 1(a) through 1(c).

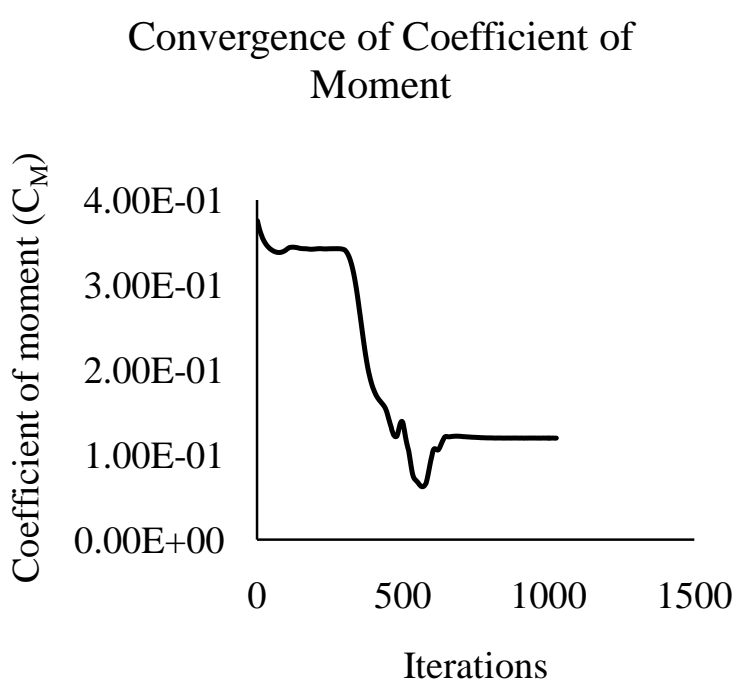

Chart -1(a): $C_{M}$ Convergence plot

Convergence of Coefficient of lift

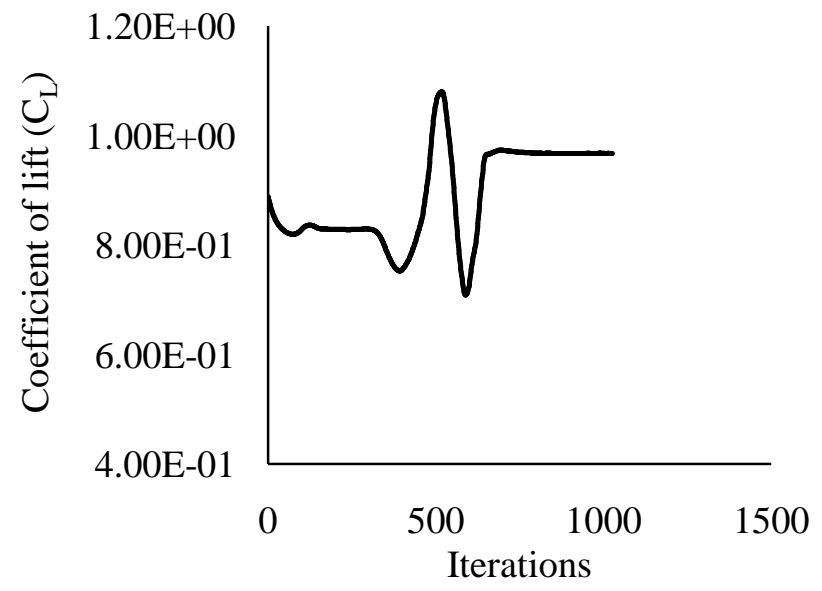

Chart -1(b): $\mathrm{C}_{\mathrm{L}}$ Convergence plot 


\section{Convergence of Coefficient of drag}

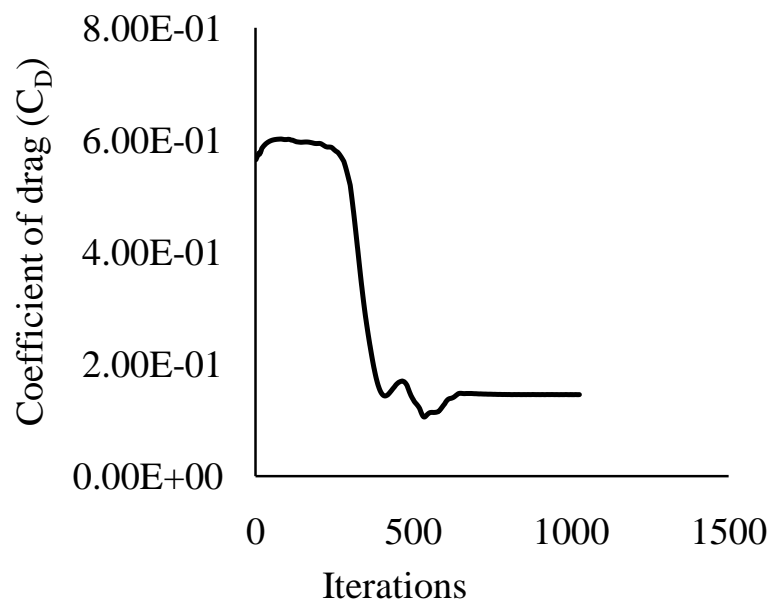

Chart -3(c): $C_{D}$ Convergence plot

\section{RESULTS}

In the following section, findings and values of variables such as coefficients of lift, drag and moment and their variations with the angles of attack have been discussed. I have attempted to discuss findings and values using some fundamental equations of fluid dynamics. As the purpose of this study was to analyze the suitability of the selected airfoil for use in a UAV, I attempted to analyze whether the graphs for the selected airfoil were following similar trend to that of the standard Global Hawk airfoil. After a reasonable amount of similarity was found between enough parameters, further it is concluded that the NACA 4415 is indeed suitable for use in a UAV.

\subsection{Distributions of Pressure}

Figures 4(a) to4(f) show the contour plot of pressure distributions across the airfoil. The colours towards the blue end of the spectrum signify regions of low pressure, whereas the colours towards the red end of the spectrum signify regions of high pressure. As the angle of attack increases the region of maximum pressure shifts to under the airfoil implying higher lift generation.

\section{$\begin{array}{ll}\text { (a) NASA LRN } 1015 & \text { (b) NACA } 4415\end{array}$}

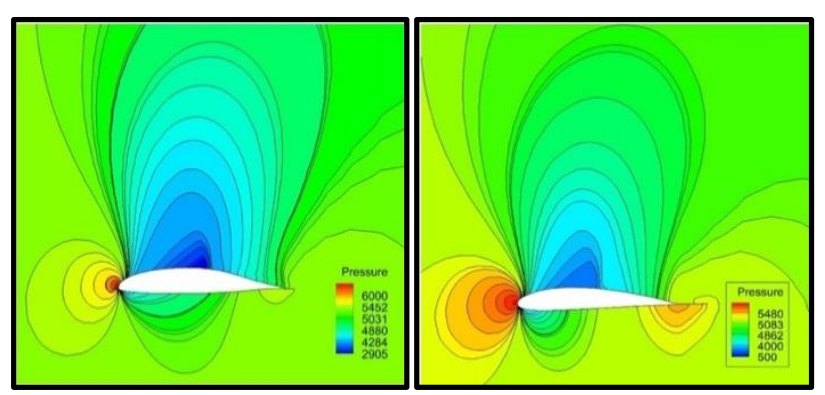

Fig -4(a):Pressure distribution $\left(0^{\circ} \mathrm{AOA}\right)$

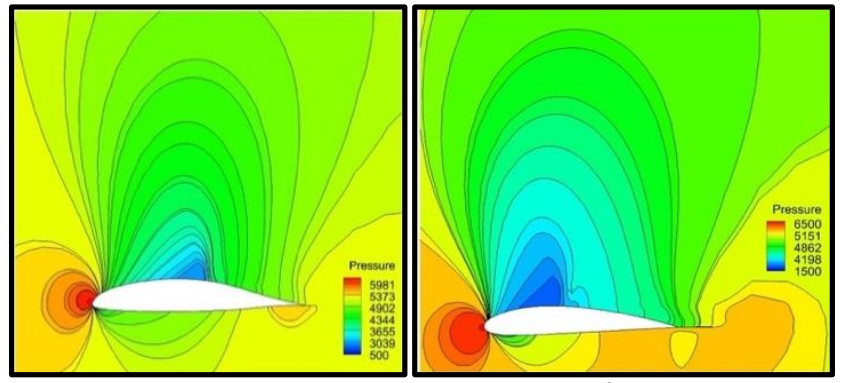

Fig -4(b): Pressure distribution ( $\left.4^{\circ} \mathrm{AOA}\right)$

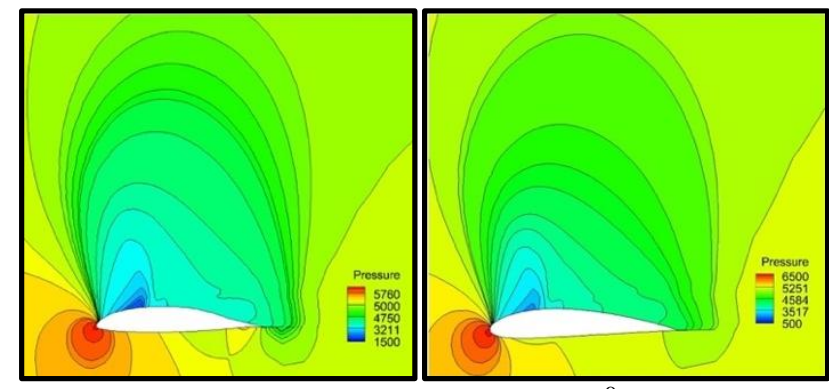

Fig -4(c): Pressure distribution ( $\left.8^{\circ} \mathrm{AOA}\right)$

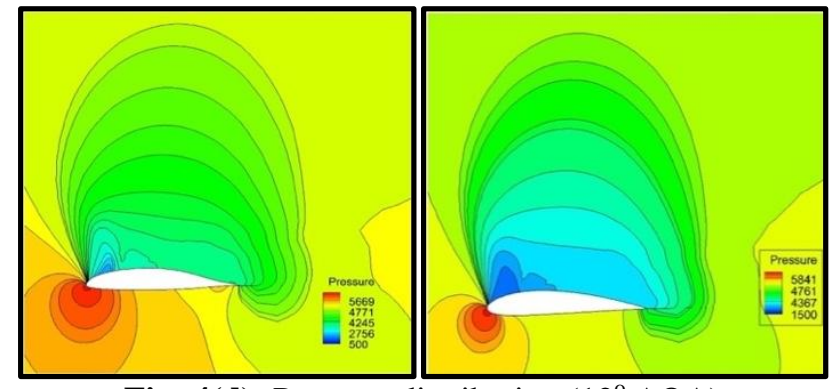

Fig -4(d): Pressure distribution (12 $\mathrm{AOA})$

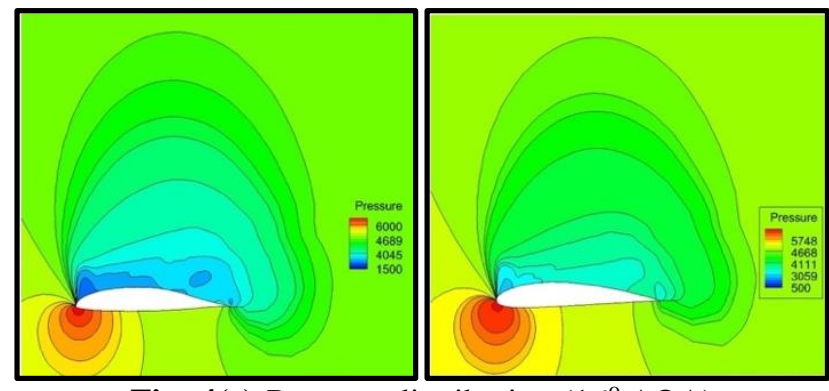

Fig -4(e):Pressure distribution $\left(16^{\circ} \mathrm{AOA}\right)$

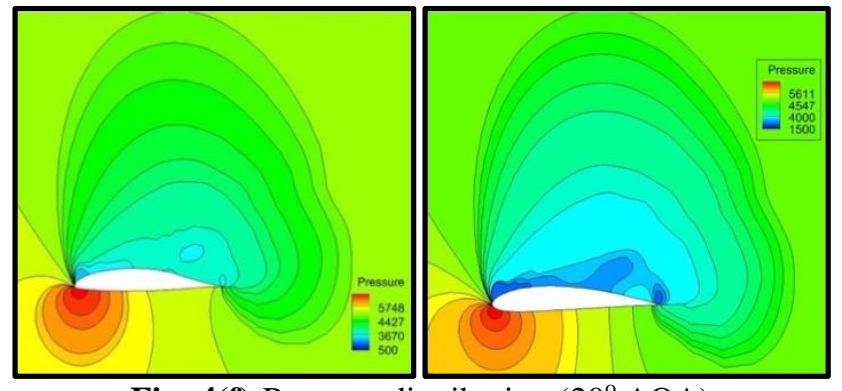

Fig -4(f):Pressure distribution $\left(20^{\circ} \mathrm{AOA}\right)$ 


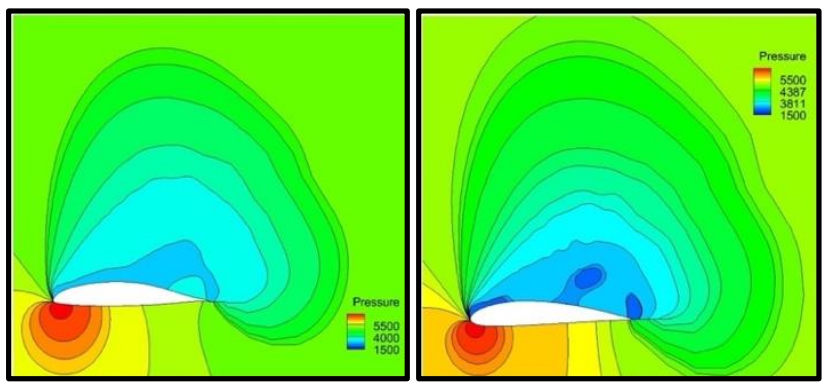

Fig -4(e):Pressure distribution $\left(24^{\circ} \mathrm{AOA}\right)$

From the pressure contours plot, it is observed region of high pressure at the leading edge (stagnation point) and low pressure region is noticed on the upper surface of the airfoil. This satisfies the Bernoulli equation theory, which states that whenever there is high velocity, we have low pressure and vice versa.

\subsection{Coefficients of Pressure}

Charts 2(a) to 2(c) shows the coefficients of pressure $(\mathrm{Cp})$ graph at upper and lower surface of the airfoil with varying angle of attacks $0^{\circ}, 12^{\circ}$ and $24^{\circ}$ respectively. It is observed that $\mathrm{Cp}$ value on the lower surface of the airfoil is greater than the incoming flow stream and as an outcome, it effectively "pushed" airfoil upward, which is normal to incoming flow stream.

Distribution of coefficient of pressure at $0^{\circ}$ Angle of attack

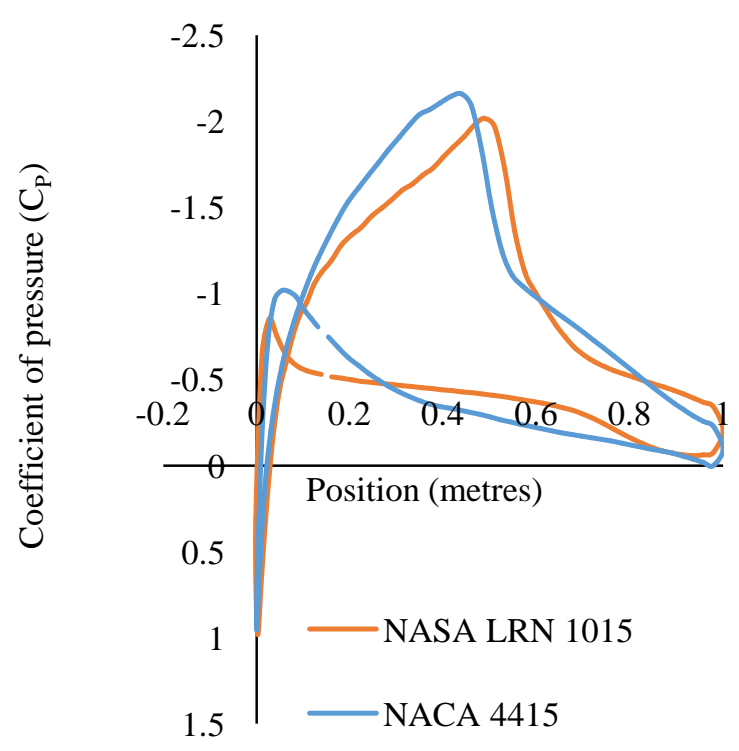

Chart -2(a): $\mathrm{C}_{\mathrm{P}}$ distribution at $0^{\circ}$

From the charts, we can see that both the airfoils captured the position and strength of the shocks correctly. The suction pressure at the leading edge is also captured fairly by both the airfoils. A minimal difference in results is noticed for both the airfoils at the trailing edge which can be due to turbulence at the trailing edge.
Distribution of coefficient of pressure at $12^{\circ}$ Angle of attack

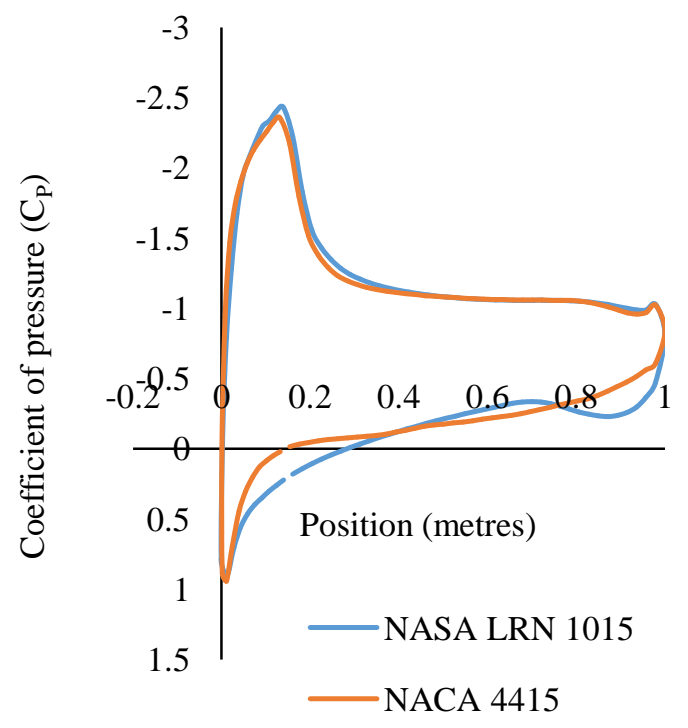

Chart -2(b): $\mathrm{C}_{\mathrm{P}}$ distribution at $12^{\circ}$

Distribution of coefficient of pressure at $24^{\circ}$ Angle of attack

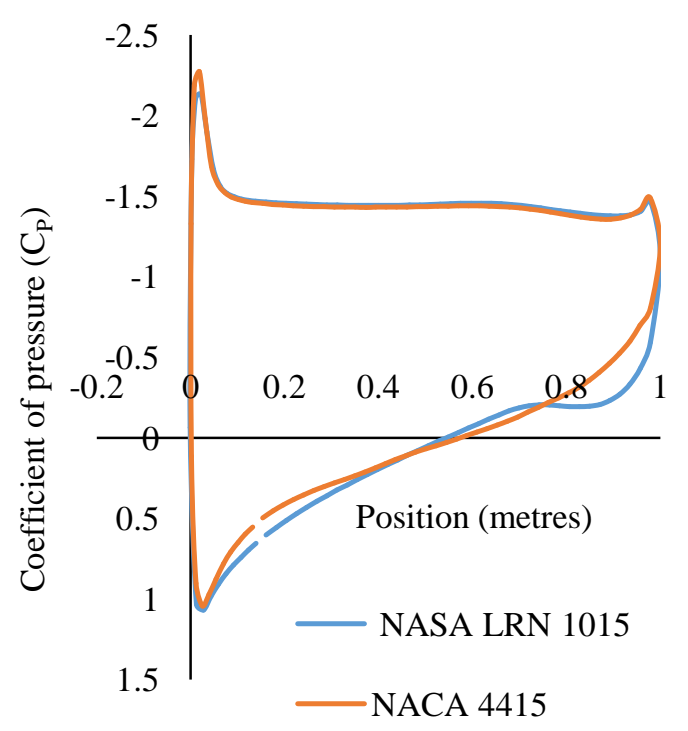

Chart -2(c): $\mathrm{C}_{\mathrm{P}}$ distribution at $24^{\circ}$

The distribution of coefficients of pressure for NACA 4415 seems to match that for NASA LRN 1015 fairly well for all three angles of attack implying that pressure distributions around NACA 4415 might make it adequate for use in a UAV. Both the results are in good agreement and overlapping with each other on upper and lower surface of airfoils.

The distribution of the respective coefficients of pressure at $0^{\circ}$ angle of attack shows that the pressure that the leading edge initially is a high positive number. This number stay fairly constant for subsequent angles of attack too. The largest negative value of the respective coefficients of 
pressure also stays almost constant for all angles of attack. However, the position of region of lowest pressure shifts from about midpoint of the chord to almost the leading edge. This implies that the maximum lift generated by both airfoils increases with increase in angle of attack making the airfoil useful not only for cruising but also to achieve altitude efficiently.

\subsection{Distribution of Mach Numbers}

Figures 6(a) through 6(g) show the contour plots of Mach number distributions across the airfoil at different angle of attacks. The colours towards the blue end of the spectrum signify regions of low Mach number, whereas the colours towards the red end of the spectrum signify regions of high Mach number. As the angle of attack increases the region of maximum pressure shifts to under the airfoil implying higher lift generation. The red regions signify a Mach number greater than 1 and the generation of shock waves in the airfoil structure. Initially, the shockwaves are concentrated on the top of the airfoil but as the angle of attack increases the shockwaves move towards the leading edge of the airfoil. The blue regions signifying region of very low Mach number signifying flow separation are seen at the trailing edge at all angles of attack. However, the flow separation is dramatically larger at higher angles of attack which is due to formation of eddies. The combination of the shock waves and flow separation may make the airfoil unstable at higher angles of attack.

(a) NASA LRN 1015

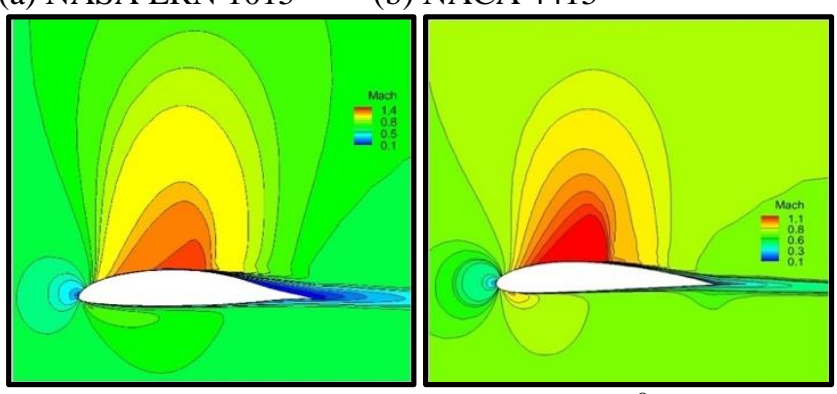

Fig -5(a): Mach number distribution $\left(0^{\circ} \mathrm{AOA}\right)$
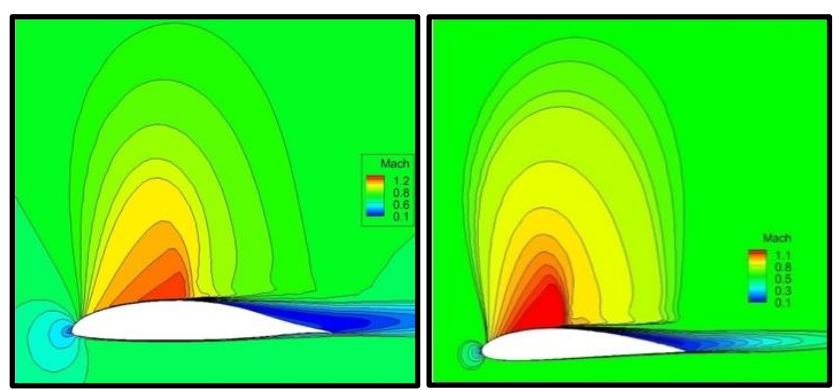

Fig -5(b): Mach number distribution $\left(4^{\circ} \mathrm{AOA}\right)$

A good agreement is noticed for both the airfoils at all angle's of attack. Their contour plots are fairly matching with each other.

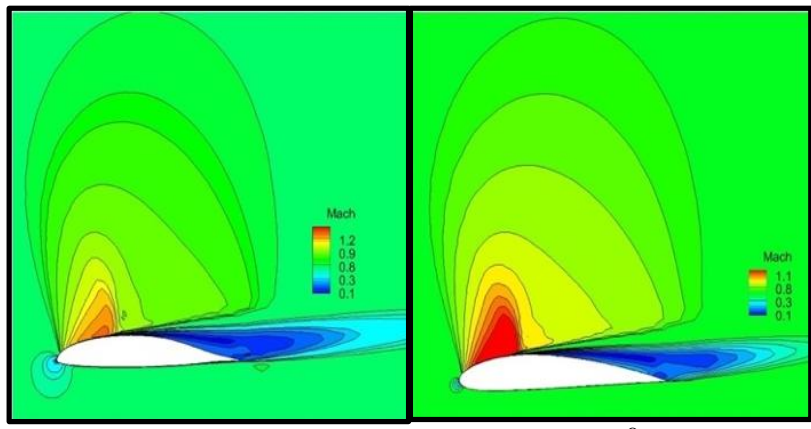

Fig -5(c): Mach number distribution $\left(8^{\circ} \mathrm{AOA}\right)$

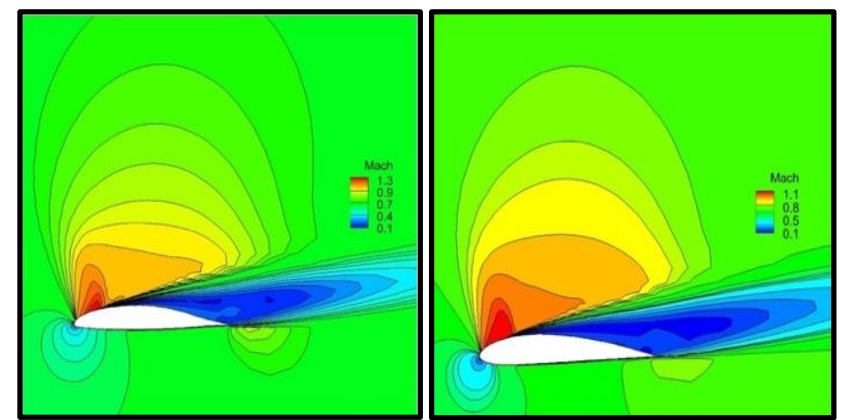

Fig -5(d): Mach number distribution $\left(16^{\circ} \mathrm{AOA}\right)$

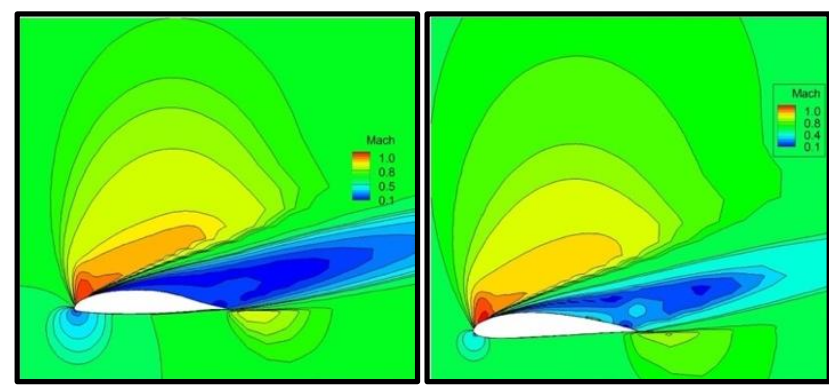

Fig -5(e): Mach number distribution $\left(16^{\circ} \mathrm{AOA}\right)$

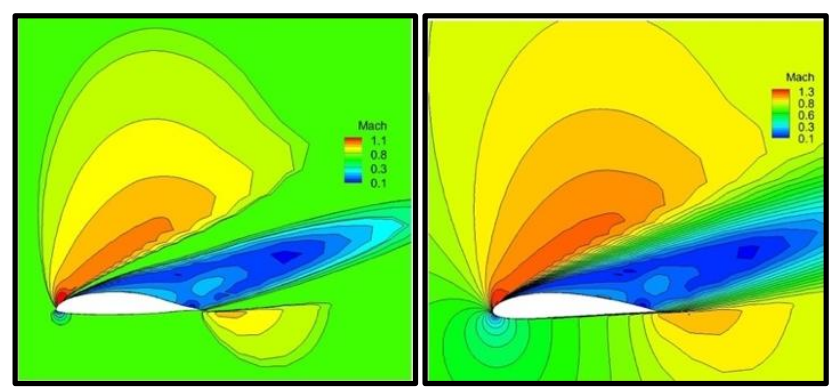

Fig -5(f): Mach number distribution $\left(20^{\circ} \mathrm{AOA}\right)$

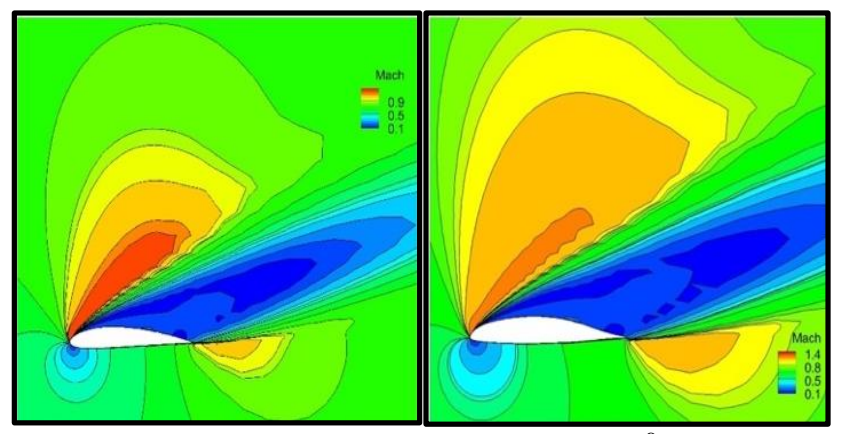

Fig -5(g):Mach number distribution $\left(24^{\circ} \mathrm{AOA}\right)$ 


\subsection{Coefficients of Lift and Drag}

Charts 3(a) and 3(b) shows the graph of Coefficient of lift $\left(C_{L}\right)$ vs angle of attack and Coefficient of drag $\left(C_{D}\right)$ Vs angle of attack respectively at different angle of attacks. Coefficient of lift increases and drag decreases as angle of attack increases for both airfoils.

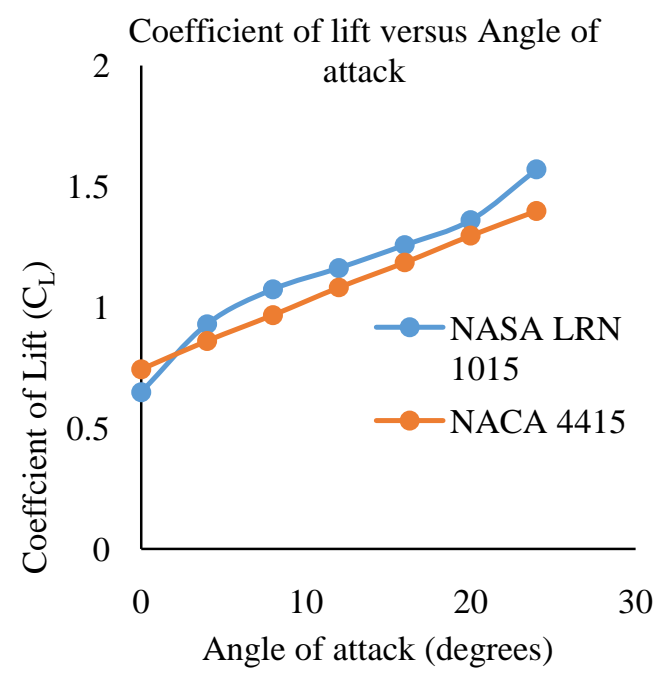

Chart -3(a): $C_{L}-$ Angle of attack distribution

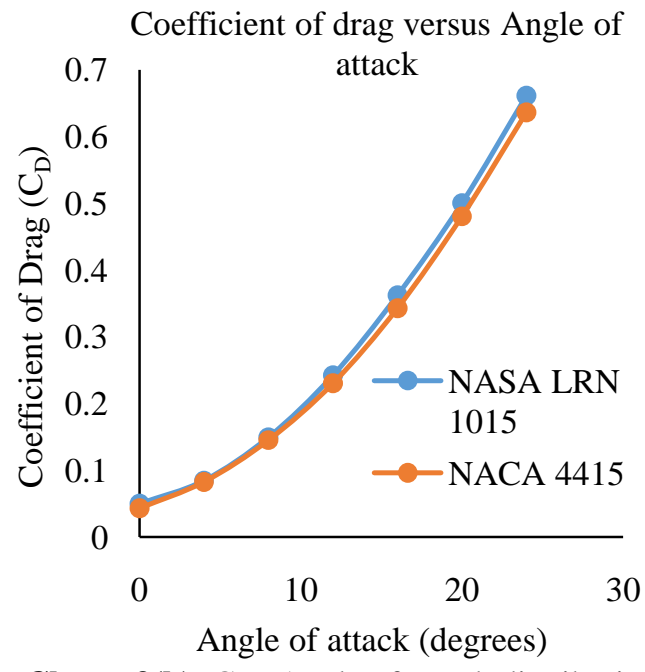

Chart -3(b): $\mathrm{C}_{\mathrm{D}}-$ Angle of attack distribution

Although initial coefficient of lift for the NACA 4415 airfoil is higher than that for NASA LRN 1015, it is consistently lower for all angles of attack other than $0^{\circ}$. Comparing the pressure distributions for both airfoils in section 5.1 at this angle of attack it is found that the pressure above the NACA 4415 airfoil drops to about 5 times less than that above the NASA LRN 1015 airfoil. This higher difference in pressure between the top and bottom surfaces leads to the high initial lift coefficient for NACA 4415.

$\mathrm{C}_{D}-\alpha$ curves for both airfoils follow similar trendsand results are completely overlapping each other, however the drag experienced by the NACA 4415 airfoil is marginally lesser. Comparing the Mach number distributions of both airfoils in section 5.3 it is seen that the region of low Mach number $(\sim 0.1)$ signifying turbulent wakes is slightly lesser for NACA 4415 airfoil. The reduction in these wakes reduces the skin drag experienced by the NACA 4415 airfoil.

\subsection{Drag Polars}

Chart 4depicts the graph for drag polar, it is plotted between Coefficient of lift $\left(C_{L}\right)$ Vs Coefficient of drag $\left(C_{D}\right)$ ngle of attack The drag polar for an airfoil is the curve depicting the relationship between the coefficients of lift and drag. As the lift generated by an airfoil increases with the angle of attack the skin drag acting on it increases as well. It is important to examine the tradeoff between these two factors to understand their net effect.

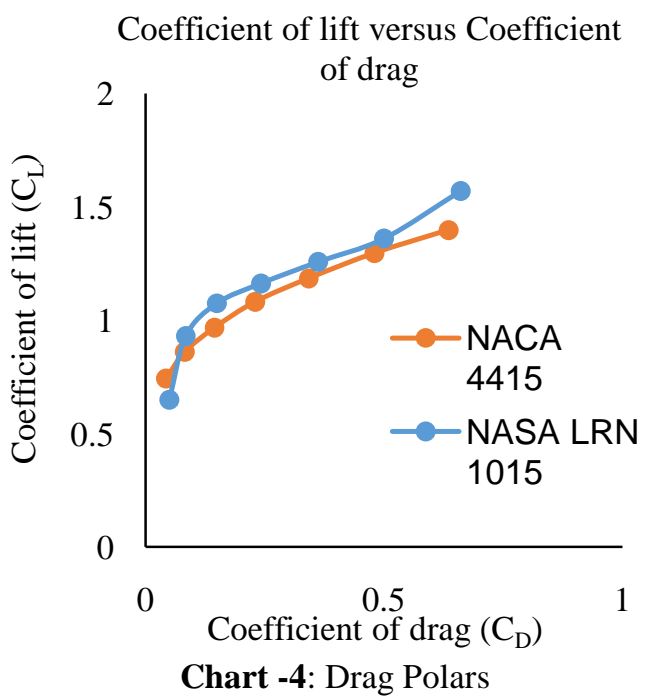

The drag polar curve for the NACA 4415 is initially higher. This is due to the higher lift generated by NACA 4415 at $0^{\circ}$ angle of attack (as explained in section 5.4).

Thereafter the drag polar for NACA 4415 decreases to a value slightly below that of the standard airfoil for each angle of attack.

Comparing the Mach number distributions for both airfoils, this trend is substantiated by the higher and more widely spread shockwave formation (indicated by regions with Mach number higher than 1) leading to higher skin drag at subsequent angles of attack for NACA 4415 than for NASA LRN 1015. This increased skin drag for similar amounts of lift generated leads to a higher drag polar for NASA LRN 1015 .

\subsection{Coefficients of Pitching Moment and Lift}

Charts 5(a) and 5(b) shows the graph of Coefficient of moment $\left(\mathrm{C}_{\mathrm{M}}\right)$ Vs angle of attack and Coefficient of moment $\left(C_{M}\right)$ Vs Coefficient of lift $\left(C_{L}\right)$ respectively at different angle of attacks. The pitch of an aircraft is the up and down movement of the nose of the aircraft and is controlled by the elevator attached at the tail of the aircraft. 


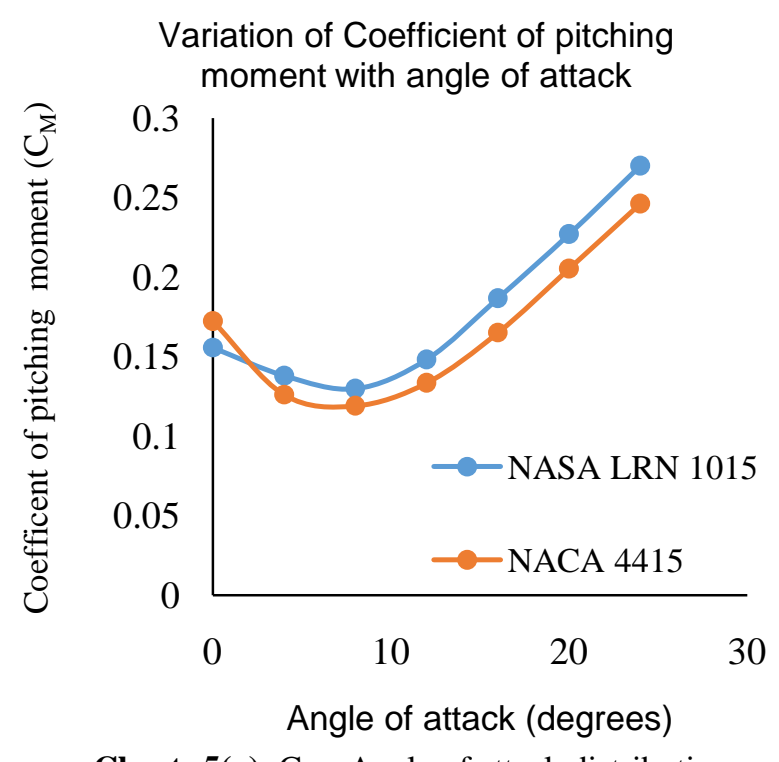

Chart -5(a): $\mathrm{C}_{\mathrm{M}}-$ Angle of attack distribution

The moment associated with this pitch along the aerodynamic center of the aircraft is known as the pitching moment of the aircraft. A corollary thus indicates that such a pitching moment can also be defined for an airfoil. The coefficient of pitching moment $\left(\mathrm{C}_{\mathrm{M}}\right)$ gives us valuable insight into the stability of the aircraft.

A positive value for coefficient of pitching moment indicates a tendency of the aircraft to tilt up whereas a negative value indicates its tendency to tilt down.

Extending this to airfoils, the graphs for both airfoils indicate an increasing tendency to tilt upwards as slopes of both graphs become increasingly positive for increasing values of angle of attack.

The coefficient of pitching moment of NACA 4415 is higher initially than that of NASA LRN 1015.This may be due to the higher lift forces at $0^{\circ}$ angle of attack (as discussed in section 5.4) generating a higher moment about the aerodynamic center causing the airfoil to tip upwards.

Coefficient of pitching moment versus Coefficient of lift

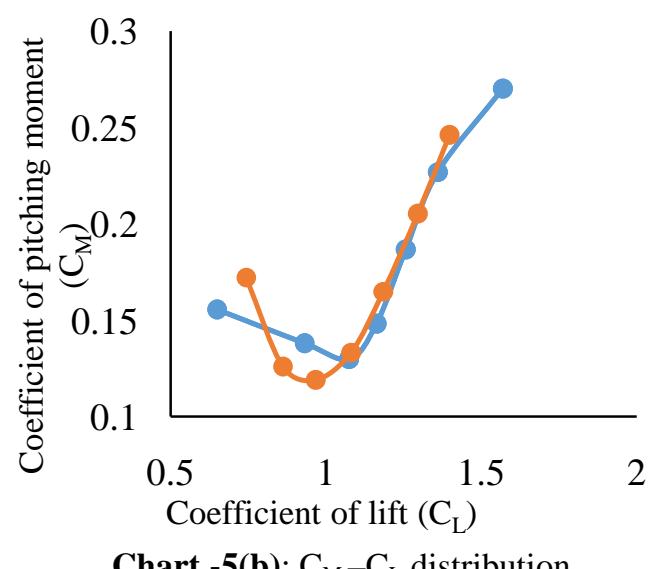

Chart -5(b): $C_{M}-C_{L}$ distribution
Further coefficients of pitching moments for NACA 4415 are lower than those for NASA LRN 1015 which may in turn be due to lower lift forces at subsequent angles of attack generating a lower moment about the aerodynamic center.

A negative slope of the variation of coefficients of pitching moment with coefficients of lift indicates positive stability whereas a positive slope indicates negative stability.

Although most commercial aircraft require positive stability, the negative stability of combat and surveillance aircraft allows them to fly at far higher speeds and increases maneuverability.

The curves of this variation for both airfoils have negative values initially indicating positive stability. However, the curve of this variation for NACA 4415 has a more negative slope that for NASA LRN 1015, indicating that it has higher stability. This may decrease maneuverability of the airfoil and thus the UAV where it is used but also increases safety.

After the initial negative slopes, the slopes for both curves become almost equivalent high positive values which indicate high instability resulting in high maneuverability and speed. This is corroborated by the Mach number distributions for both airfoils where at higher angles of attack (and thus higher lift as explained in section 5.4) the shockwave formation decreases to a small region allowing the airfoil to maneuver and accelerate without experiencing too much skin drag.

\section{CONCLUSION}

In this paper, aerodynamic parameters, flow behavior around airfoils NACA 4415 and NASA LRN 1015 were studied and analyzed in depth. The CFD results for both the airfoils show good agreement with each other. The results also show similar trends for both airfoils. It is concluded, thus, that the NACA 4415 is indeed suitable for use in a UAV.

\section{ACKNOWLEDGEMENT}

The authors can acknowledge any person/authorities in this section. This is not mandatory.

\section{REFERENCES}

[1]. National Aeronautics and Space Administration. (2015, May 05). Retrieved August 2016, from grc.nasa.gov: https://www.grc.nasa.gov/www/k-12/airplane/wrong1.html [2]. National Aeronautics and Space Administration. (2015, May 05). Retrieved August 2016, from grc.nasa.gov: https://www.grc.nasa.gov/www/k-12/airplane/bernnew.html [3]. Şahin, İ, \&Acir, A. (2015, February). Numerical and Experimental Investigations of Lift and Drag Performances of NACA 0015 Wind Turbine Airfoil. International Journal of Materials, Mechanics and Manufacturing, 3(1), 22-25.

[4]. Eleni, D. C., Athanasios, T. I., \&Dionissios, M. P. (2012, March). Evaluation of the turbulence models for the simulation of the flow over a National Advisory Committee 
for Aeronautics (NACA) 0012 airfoil. Journal of Mechanical Engineering Research, 4, 100-111.

[5]. Kevadiya, M. (2013, May). CFD Analysis of Pressure Coefficient for NACA 4412. International Journal of Engineering Trends and Technology, 4(5), 2041-2043.

[6]. Gulzar, O., Gulzar, S., Bhatele, S., \&Soni, N. (2014). Impact of Variation in Angle of Attack on NACA 7420 Airfoil in Transonic Compressible Flow Using SpalartAllmaras Turbulence Model. International Journal of Research in Mechanical Engineering and Technology, 4 (2), 35-36.

[7]. Marathe, M. S., \& Bansode, S. N. (2013). Airfoil Selection of MAV (Miniature Air Vehicle) for Low Reynolds Number. nternational Journal on Theoretical and Applied Research in Mechanical Engineering, 2 (4), 38-42.

[8]. Hossain, M. S., Raiyan, M. F., Akanda, M. N., \& Jony, N. H. (2014). A COMPARATIVE FLOW ANALYSIS OF NACA 6409 AND NACA 4412 AEROFOIL . International Journal of Research in Engineering and Technology, 3 (10), 342-350.

[9]. Ahmed, T., Amin, M. T., Islam, S. R., \& Ahmed, S. (2013). Computational Study of Flow Around a NACA 0012 Wing Flapped at Different Flap Angles with Varying Mach Numbers. Global Journal of Researches in Engineering General Engineering, 13 (4), 5-15.

[10]. http://airfoiltools.com/airfoil/details?airfoil=lrn1015il. (n.d.). Retrieved January 2017, from airfoiltools: http://airfoiltools.com

\section{BIOGRAPHIES}

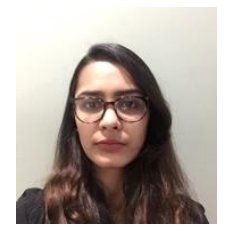

Anjali Balani,a second year student at the University of Michigan, Ann Arbor, is pursuing an undergraduate degree in aerospace engineering. Former engineering apprentice at Hindustan Aeronautics Limited (HAL), Nasik division.

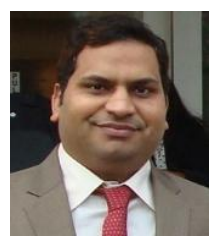

Tarun Yadav, working as Manager at Design Armament Department, Aircraft Upgrade Research and Design Centre (AURDC), Hindustan Aeronautics Limited (HAL), Nasik Division. Degree holder of Master of Science (M.Sc) in Computational Fluid Dynamics from Cranfield University, United Kingdom.

Contact details: E-mail ID: tarun_ietru@yahoo.co.in

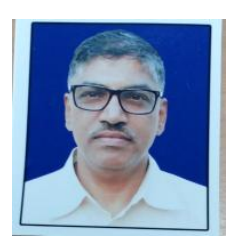

Appasaheb Malagaudanavar, working as AGM, Aircraft Upgrade Research and Design Centre (AURDC), Hindustan Aeronautics Limited (HAL), Nasik Division. Degree holder of Master of Technology (M.Tech) from Indian Institute of Technology, Madras, India 